\title{
Correlation of Primary Spontaneous Pneumothorax and Air Pollution in Adolescents
}

\author{
Byung Mo Gu, M.D. ', Ho Hyun Ko, M.D. ', Yong Joon Ra, M.D. ', Hee Sung Lee, M.D., Ph.D. ${ }^{\text {, }}$ \\ Hyoung Soo Kim, M.D., Ph.D. ${ }^{1}$, Hong Kyu Lee, M.D. ${ }^{1}$ \\ 'Department of Thoracic and Cardiovascular Surgery, Hallym University Sacred Heart Hospital, Anyang; ${ }^{2}$ Department of Thoracic and Cardiovascular Surgery, \\ Hallym University Dongtan Sacred Heart Hospital, Hwaseong, Korea
}

\section{ARTICLE INFO}

Received June 10, 2020

Revised September 25, 2020

Accepted October 4, 2020

Corresponding author

Hong Kyu Lee

Tel $82-31-380-3815$

Fax 82-31-380-4118

E-mail hklee0228@hallym.or.kr

ORCID

https://orcid.org/0000-0002-9087-7783

\begin{abstract}
Background: We aimed to investigate the characteristics of primary spontaneous pneumothorax (PSP) in adolescents and to analyze the relationship between the occurrence of PSP and air pollutants.

Methods: Data pertaining to age, sex, body mass index, smoking status, initial pneumothorax volume, presence of bullae, treatment methods, and city of residence were retrospectively obtained from January 2010 to December 2014. We investigated the association between short-term exposure to air pollutants $\left(\mathrm{SO}_{2}, \mathrm{NO}_{2}, \mathrm{O}_{3^{\prime}} \mathrm{CO}\right.$, and $\left.\mathrm{PM}_{10}\right)$ and the occurrence of PSP using a case-crossover design with conditional logistic regression.

Results: We collected information from 598 patients who were admitted for PSP, with a mean follow-up duration of 62.9 months. The majority (91.1\%) of the patients were male. In the case-crossover design, conditional logistic regression showed that no air pollutant was associated with the occurrence of pneumothorax. The results were consistent across all city subgroups (Anyang, Gunpo, Uiwang, and Gwacheon).

Conclusion: In our study, the incidence rate of pneumothorax was 153.8 per 100,000 person-years in male adolescents and 16.7 per 100,000 person-years in female adolescents. The case-crossover design showed that PSP in adolescents is unlikely to be related to air pollution.
\end{abstract}

Keywords: Pneumothorax, Air pollution, Adolescent, Epidemiology

\section{Introduction}

Pneumothorax is an accumulation of air in the pleural space that leads to lung collapse. Primary spontaneous pneumothorax (PSP) is pneumothorax without known lung disease, while secondary spontaneous pneumothorax occurs in people with underlying lung diseases, such as chronic obstructive pulmonary disease and congenital lung disease. Traumatic pneumothorax results from damage to lung tissue, such as rib fractures or injuries caused by needles. It can also be caused by blunt chest trauma without direct penetration of the lung. PSP does not pose a high risk for mortality and morbidity, but its suddenly-occurring nature, requirement of hospitalization for treatment, and high recurrence rate (32\% according to a meta-analysis) incur high socioeconomic costs [1]. Therefore, several studies have been conducted on the pathophysiology and epidemiology of PSP. It is well known from these studies that PSP occurs more frequently in younger people with a low body mass index (BMI), and it shows a clear male predilection [2]. The incidence rate is slightly different in each country, ranging from 17 to 24 to per 100,000 person-years in men and from 1 to 6 per 100,000 person-years in women [3].

The cause of PSP is thought to be the rupture of small, peripheral, usually apical subpleural blebs. Smoking is the most important risk factor for the development of PSP, as it is known to increase the incidence of PSP by 9-22 times [4]. In connection with smoking, meteorological factors and air pollutants have been noted as elements involved in the rupture of bullae in the past. Some studies have investigated this relationship, but with inconsistent results. Bertolac- 
Table 1. Patients' characteristics

\begin{tabular}{|c|c|}
\hline Characteristic & Value \\
\hline Mean age (yr) & $17.2 \pm 1.49$ \\
\hline Male & $545(91.1)$ \\
\hline Female & $53(8.9)$ \\
\hline Height (cm) & $173.9 \pm 6.51$ \\
\hline Weight (kg) & $58.2 \pm 8.36$ \\
\hline Body mass index $\left(\mathrm{kg} / \mathrm{m}^{2}\right)$ & $19.2 \pm 2.19$ \\
\hline Smoking & 47 (7.9) \\
\hline \multicolumn{2}{|l|}{ Location } \\
\hline Left side & $339(56.7)$ \\
\hline Right side & $251(42.0)$ \\
\hline Bilateral & $8(1.3)$ \\
\hline \multicolumn{2}{|l|}{ Size } \\
\hline Small & $354(59.2)$ \\
\hline Large & $230(38.5)$ \\
\hline Bulla & $289(48.3)$ \\
\hline Ipsilateral side & $173(59.9)$ \\
\hline Contralateral side & $2(0.7)$ \\
\hline Both sides & $114(39.4)$ \\
\hline \multicolumn{2}{|l|}{ Treatment } \\
\hline $\mathrm{O}_{2}$ management & $8(1.3)$ \\
\hline Chest tube management & $172(28.8)$ \\
\hline Operation & $418(69.9)$ \\
\hline Length of stay (day) & $6.6(1-31)$ \\
\hline
\end{tabular}

Values are presented as mean \pm standard deviation, number $(\%)$, or mean (range), unless otherwise stated.

cini et al. [5] reported that temperature, wind speed, and concentration of air pollutants might explain clusters in hospitalization for PSP. Moreover, in a study conducted by Han et al. [6] in Seoul, Korea, particulate matter (PM) and carbon monoxide $(\mathrm{CO})$ were identified as possible predisposing factors for PSP, while another study argued that ozone $\left(\mathrm{O}_{3}\right)$ and nitrogen dioxide $\left(\mathrm{NO}_{2}\right)$ were significantly associated with PSP [7]. However, a case-crossover study in France failed to show a relationship between PSP and short-term air pollution exposure [8].

In the present study, we aimed to examine the association between PSP and air pollutants in adolescents who had no underlying lung disease and either had never smoked or had a negligible smoking history. Although PSP is known to occur in young people, its incidence rate in adolescents has not been clearly studied [9]. Therefore, based on the information obtained in this study, the incidence rate of adolescent pneumothorax will also be investigated.

\section{Methods}

A single-center retrospective study was conducted of the

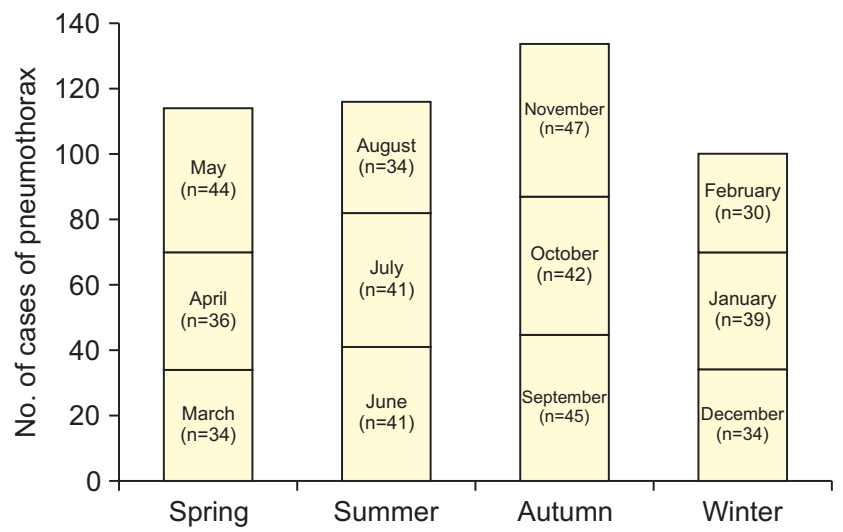

Fig. 1. Seasonal and monthly trends in pneumothorax incidence.

medical records of 632 PSP admissions in adolescents, defined as between 10 and 19 years of age at the time of admission, from January 2010 to December 2014. Among these episodes, we excluded patients with underlying diseases such as pneumonia, tuberculosis, asthma, and other congenital or genetic lung diseases. We also excluded cases of traumatic or iatrogenic pneumothorax and spontaneous hemopneumothorax. Ultimately, 598 patients were eligible for analysis.

Data pertaining to age, sex, BMI, smoking status, initial pneumothorax volume, bulla presence, treatment, and city of residence were retrospectively obtained from our institutional database. The Institutional Review Board of Hallym University Sacred Heart Hospital approved this study without informed consent (IRB approval no., 2020-02-004001). Most patients, with the exception of 2 , underwent chest computed tomography (CT), and we recorded the presence and location of bullae in the chest. Treatment methods were generally selected according to the British Thoracic Society (BTS) guidelines and were classified into observation with oxygen inhalation, chest tube insertion, and surgical intervention [10]. Patients were classified based on their final treatment.

Because PSP is an acute disease, most patients visited or were transported to the hospital in their vicinity. Thus, we investigated air pollutant data in 4 cities (Anyang, Gunpo, Uiwang, and Gwacheon) near the hospitals where most of the patients (93.8\%) resided. We hypothesized that if a cluster of PSP hospitalizations occurred, it would be due to weekly changes in pollutant concentrations, rather than daily changes. Therefore, we designed a case-crossover study to investigate the relationship between the occurrence of PSP and air pollution. Rather than assessing the preceding 3 to 4 days, as done by other researchers [5-8], 

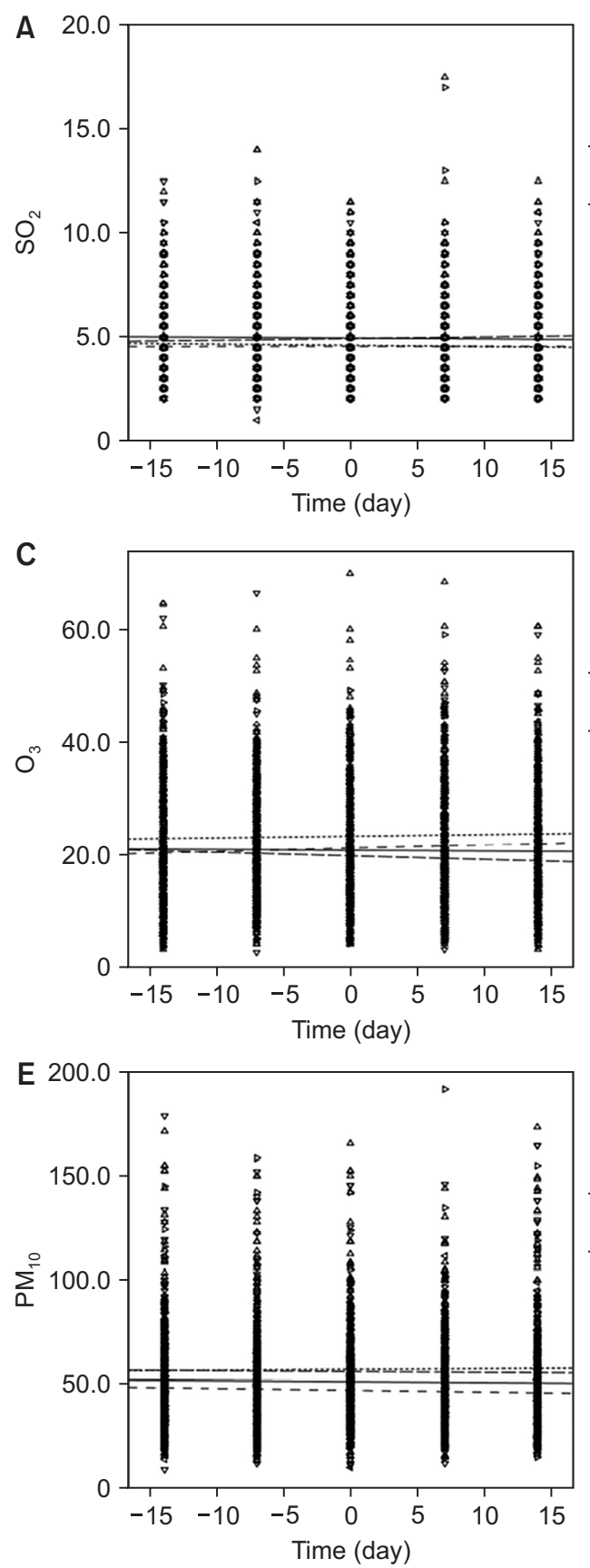

$\triangle$ Anyang

$\nabla$ Gunpo

$\triangleleft$ Gwacheon

$\triangleright$ Uiwang

- Anyang

-... Gunpo

-- Gwacheon

-- Uiwang

$\triangle$ Anyang

$\nabla$ Gunpo

$\triangleleft$ Gwacheon

$\triangleright$ Uiwang

- Anyang

-... Gunpo

- - Gwacheon

-- Uiwang

$\triangle$ Anyang

$\nabla$ Gunpo

$\triangleleft$ Gwacheon

$\triangleright$ Uiwang

- Anyang

-... Gunpo

--- Gwacheon

-- Uiwang
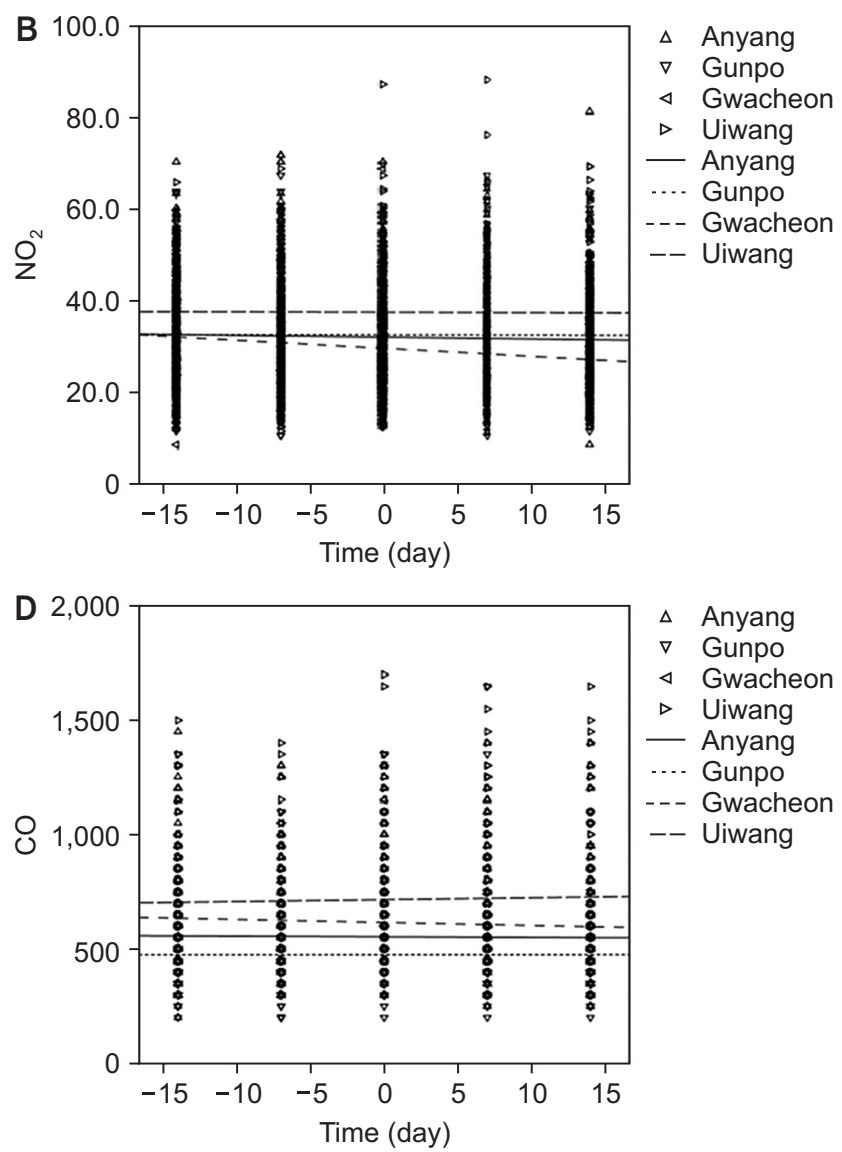

Fig. 2. Scatter plots and trendlines between $\mathrm{SO}_{2}$ concentration and time $(\mathrm{A}), \mathrm{NO}_{2}$ concentration and time $(\mathrm{B}), \mathrm{O}_{3}$ concentration and time $(\mathrm{C}), \mathrm{CO}$ concentration and time (D), and $\mathrm{PM}_{10}$ concentration and time (E). Time 0 refers to the case-day, and times -7 , $-14,+7$, and +14 refer to the days 1 week or 2 weeks before and after admission, respectively.

we collected air pollutant data at 1 and 2 weeks before and after admission, as well as on the day of admission itself, to assess the weekly effects of air pollution.

\section{Case-crossover design}

We assessed the association between short-term exposure to air pollutants $\left(\mathrm{SO}_{2}, \mathrm{NO}_{2}, \mathrm{O}_{3}, \mathrm{CO}\right.$, and $\left.\mathrm{PM}_{10}\right)$ and the occurrence of PSP using a case-crossover design [11]. First,

the case-day was defined as the date of admission due to pneumothorax. For each patient, we compared the daily air pollutant levels on the case-day to those on control days, which were defined as 1 week and 2 weeks before and after admission, so that 4 control days were selected per case-day. All data were collected according to the city of residence and statistically analyzed using conditional logistic regression. 


\section{Statistical analysis}

All categorical variables are represented as absolute figures and percentages, while continuous variables (such as $\mathrm{BMI})$ are represented as mean \pm standard deviation. All p-values $<0.05$ were considered to indicate statistical significance. Conditional logistic regression was performed using IBM SPSS ver. 24.0 (IBM Corp., Armonk, NY, USA).

\section{Results}

Of the 632 patients with PSP, 34 were excluded according to the exclusion criteria, and 598 patients were included; their characteristics are summarized in Table 1 . The mean age was 17.2 years and 545 were males (91.1\%). The mean BMI was $19.2 \mathrm{~kg} / \mathrm{m}^{2}$, which is within in the World Health Organization range of average, but towards the lower end of the category. The initial pneumothorax amount was categorized as small or large according to the BTS guideline [10]. Bullae were seen on chest CT in 289 patients (48.3\%), of whom 173 patients had a bulla on the ipsilateral side, 2 patients had a bulla on the contralateral side, and 114 patients had bullae on both sides. The treatment was mainly based on the BTS guidelines, unless the parents preferred otherwise. Only 8 patients (1.3\%) were discharged after recovery with oxygen therapy, while 172 (28.8\%) underwent chest tube insertion. The remaining 418 patients (69.9\%) underwent surgical treatment. The mean length of stay was 6.6 days.

Our institution is a tertiary referral center, and it covers most of the pneumothorax patients in the above-mentioned 4 cities (Anyang, Gunpo, Uiwang, and Gwacheon). In this study, approximately 100 males and 10 females were admitted with PSP per year. The teenager population of these 4 cities is about 125,000 , with a total of 65,000 male adolescents and 60,000 female adolescents (Korean Statistical Information Service 2014). The incidence rate of pneumothorax was 153.8 per 100,000 person-years in male adolescents and 16.7 per 100,000 person-years in female adolescents.

Based on our institutional database, we summurized the seasonal and monthly status of pneumothorax (Fig. 1). Pneumothorax most frequently occurred in November, and the most common season was autumn. The month with the lowest number of cases of pneumothorax was February, and the season with the lowest occurrence of pneumothorax was winter.

Herein, $\mathrm{SO}_{2}, \mathrm{NO}_{2}, \mathrm{O}_{3}$, and $\mathrm{CO}$ concentrations are shown as parts per thousand and $\mathrm{PM}_{10}$ concentrations as $\mu \mathrm{g} / \mathrm{m}^{3}$.
Table 2. Conditional logistic regression between air pollutants and the occurrence of pneumothorax

\begin{tabular}{|c|c|c|}
\hline Factor & p-value & $\begin{array}{c}\text { Odds ratio (95\% } \\
\text { confidence interval) }\end{array}$ \\
\hline $\mathrm{SO}_{2}$ (total) & 0.841 & $1.010(0.919-1.109)$ \\
\hline $\mathrm{NO}_{2}$ (total) & 0.692 & $0.997(0.981-1.013)$ \\
\hline $\mathrm{O}_{3}$ (total) & 0.572 & $0.996(0.981-1.011)$ \\
\hline $\mathrm{CO}$ (total) & 0.523 & $1.000(0.999-1.001)$ \\
\hline $\mathrm{PM}_{10}$ (total) & 0.841 & $0.999(0.993-1.006)$ \\
\hline $\mathrm{SO}_{2}$ (Anyang) & 0.439 & $1.051(0.927-1.192)$ \\
\hline $\mathrm{NO}_{2}$ (Anyang) & 0.373 & $0.989(0.967-1.013)$ \\
\hline $\mathrm{O}_{3}$ (Anyang) & 0.084 & $0.983(0.998-1.001)$ \\
\hline CO (Anyang) & 0.919 & $1.000(0.998-1.001)$ \\
\hline $\mathrm{PM}_{10}$ (Anyang) & 0.455 & $1.003(0.995-1.012)$ \\
\hline $\mathrm{SO}_{2}$ (Gunpo) & 0.754 & $1.032(0.848-1.255)$ \\
\hline $\mathrm{NO}_{2}$ (Gunpo) & 0.785 & $0.995(0.963-1.029)$ \\
\hline $\mathrm{O}_{3}$ (Gunpo) & 0.663 & $0.992(0.959-1.027)$ \\
\hline CO (Gunpo) & 0.800 & $1.000(0.997-1.002)$ \\
\hline $\mathrm{PM}_{10}$ (Gunpo) & 0.919 & $0.999(0.986-1.013)$ \\
\hline $\mathrm{SO}_{2}$ (Uiwang) & 0.402 & $0.900(0.979-1.012)$ \\
\hline $\mathrm{NO}_{2}$ (Uiwang) & 0.466 & $1.014(0.703-1.151)$ \\
\hline $\mathrm{O}_{3}$ (Uiwang) & 0.353 & $1.017(0.981-1.054)$ \\
\hline CO (Uiwang) & 0.490 & $1.001(0.999-1.003)$ \\
\hline $\mathrm{PM}_{10}$ (Uiwang) & 0.618 & $0.996(0.979-1.012)$ \\
\hline $\mathrm{SO}_{2}$ (Gwacheon) & 0.371 & $0.827(0.545-1.254)$ \\
\hline $\mathrm{NO}_{2}$ (Gwacheon) & 0.755 & $1.014(0.929-1.106)$ \\
\hline $\mathrm{O}_{3}$ (Gwacheon) & 0.076 & $1.068(0.993-1.148)$ \\
\hline CO (Gwacheon) & 0.058 & 1.005 (1.000-1.011) \\
\hline $\mathrm{PM}_{10}$ (Gwacheon) & 0.076 & $0.996(0.930-1.004)$ \\
\hline
\end{tabular}

The number of patients in each city was 331 from Anyang, 110 from Gunpo, 88 from Uiwang, 31 from Gwacheon, and 38 from other regions. After exclusion of the 38 patients from other regions, the relationships between the air pollutant data in each city and the date are shown in a scatter plot (Fig. 2). The trendlines are shown in each figure, indicating that the 2 variables had a very weak relationship (coefficient of determination $<0.01$ ).

In the case-crossover design, the conditional logistic regression results are shown in Table 2. No air pollutant was associated with incident pneumothorax. The same results were obtained in a subgroup analysis of participants from each city (Anyang, Gunpo, Uiwang, and Gwacheon).

\section{Discussion}

The incidence rate of pneumothorax in the general population is $17-24$ per 100,000 person-years in men and 1-6 per 100,000 person-years in women [3]. Our figure (153.8 per 100,000 person-years in male adolescents and 16.7 per 100,000 person-years in female adolescents) is surprisingly 
high when compared with previous studies of the general population. The actual incidence rate may even be much higher because this figure does not include patients who visited hospitals in other regions and with asymptomatic small pneumothorax who did not come to our hospital. This figure is shockingly high, even considering the frequent occurrence of pneumothorax in adolescents; therefore, further research is needed based on the national medical database.

We classified patients according to the final treatment method, and 418 patients (69.9\%) eventually underwent surgical intervention. This is thought to reflect the reduced burden of surgery owing to the development of minimally invasive video-assisted thoracoscopic surgery. It also shows a tendency for adolescent patients and their parents to prefer surgery due to their fears of recurrence, which may be unpredictable.

Pneumothorax most frequently occurred in November, while the month with the lowest number of pneumothorax cases was February. Lee et al. [12] also studied PSP in adolescents and showed exactly the same results for monthly occurrence. A possible reason for this finding is the increase in outdoor activities. However, further research is required to explore this possibility by deploying appropriate statistical techniques.

The correlation between the occurrence of pneumothorax and air pollution remains unclear. In particular, it is not known how much time is required for air pollutants to cause pneumothoax. Previous studies investigated the relationship between short-term exposure to air pollution and the occurrence of pneumothorax by examining air pollution levels 1 to 3 days before the onset of pneumothorax, but found inconsistent results [5-8]. Based on the discrepancies in previous results, we questioned the hypothesis that pneumothorax is caused by short-term (1-3 days) exposure to air pollution. Therefore, we focused on the patterns of onset of pneumothorax. Smit et al. [13] argued for the first time that pneumothorax occurs like mini-epidemics, in which patients with spontaneous pneumothorax are admitted within 3 days of each other. Although other studies reported inconsistent results regarding the cause of pneumothorax, they consistently found that pneumothorax occurred in clusters $[5,7,14]$. Based on this fact, we explored the hypothesis that the relationship between pneumothorax and air pollution could be affected by weekly changes rather than daily changes.

A major strength of this study is that it included adolescents with a relatively healthy lung parenchyma. In contrast, adults may have lung damage due to cigarette smok- ing or occupational factors. In the study population, the total smoking rate was only 49 patients $(7.9 \%)$ and their smoking history was not remarkable (less than 0.5 packyear). Therefore, this population was suitable for assessing the relationship between pneumothorax and air pollution, while excluding other factors. To the best of our knowledge, this is the first study to address the relationship between weekly changes in air pollution and pneumothorax.

The main limitation of this study is that since it was a retrospective study based on medical records, it could not account for differences in the degree and time of exposure to air pollution in each patient. In addition, this study included both emergency department patients and outpatients. Unlike patients in the emergency department, those who present to an outpatient clinic usually have mild symptoms and therefore tend to come several days after the onset of pneumothorax, which might have affected the results.

Our study investigated the characteristics of PSP in adolescents and the relationship between outbreaks of PSP in adolescents and air pollutants. In our study, the incidence rate of pneumothorax was 153.8 per 100,000 person-years in male adolescents and 16.7 per 100,000 person-years in female adolescents. From this case-crossover design, we found that PSP in adolescents was not associated with weekly changes in air pollution. Further research needs to consider different study designs to identify causes of the cluster hospitalization pattern of pneumothorax.

\section{Conflict of interest}

No potential conflict of interest relevant to this article was reported.

\section{Acknowledgments}

We would like to thank the Korean Statistical Information Service for their data support on air pollution and population.

\section{ORCID}

Byung Mo Gu: https://orcid.org/0000-0001-9632-6803

Ho Hyun Ko: https://orcid.org/0000-0002-2612-5026

Yong Joon Ra: https://orcid.org/0000-0003-2153-504X

Hee Sung Lee: https://orcid.org/0000-0002-2615-8346

Hyoung Soo Kim: https://orcid.org/0000-0001-6023-0818

Hong Kyu Lee: https://orcid.org/0000-0002-9087-7783 


\section{References}

1. Walker SP, Bibby AC, Halford P, Stadon L, White P, Maskell NA. Recurrence rates in primary spontaneous pneumothorax: a systematic review and meta-analysis. Eur Respir J 2018;52:1800864.

2. Tschopp JM, Bintcliffe O, Astoul P, et al. ERS task force statement: diagnosis and treatment of primary spontaneous pneumothorax. Eur Respir J 2015;46:321-35.

3. Savitsky E, Oh SS, Lee JM. The evolving epidemiology and management of spontaneous pneumothorax. JAMA 2018;320:1441-3.

4. Bense L, Eklund G, Wiman LG. Smoking and the increased risk of contracting spontaneous pneumothorax. Chest 1987;92:1009-12.

5. Bertolaccini L, Alemanno L, Rocco G, Cassardo C. Air pollution, weather variations and primary spontaneous pneumothorax. J Thorac Dis 2010;2:9-15.

6. Han C, Lim YH, Jung K, Hong YC. Association between ambient air pollution exposure and spontaneous pneumothorax occurrence. Epidemiology 2019;30 Suppl 1:S48-56.

7. Park JH, Lee SH, Yun SJ, et al. Air pollutants and atmospheric pressure increased risk of ED visit for spontaneous pneumothorax. Am J
Emerg Med 2018;36:2249-53.

8. Marx T, Bernard N, Parmentier AL, et al. Does air pollution really impact the onset of spontaneous pneumothorax?: a French case-crossover study. Environ Int 2019;127:317-23.

9. Furia S, Breda C. Primary spontaneous pneumothorax in children and adolescents: a systematic review. Pediatr Med 2019;2:12.

10. MacDuff A, Arnold A, Harvey J; BTS Pleural Disease Guideline Group. Management of spontaneous pneumothorax: British Thoracic Society Pleural Disease Guideline 2010. Thorax 2010;65 Suppl 2: ii18-31.

11. Di Q, Dai L, Wang Y, et al. Association of short-term exposure to air pollution with mortality in older adults. JAMA 2017;318:2446-56.

12. Lee SJ, Cha MA, Woo YR, Kwon EB, Ahn YH. Clinical characteristics of primary spontaneous pneumothorax in adolescents: factors for recurrence. Allergy Asthma Respir Dis 2014;2:251-8.

13. Smit HJ, Deville WL, Schramel FM, Postmus PE. Spontaneous pneumothorax: predictable mini-epidemics? Lancet 1997;350:1450.

14. Alifano M, Forti Parri SN, Bonfanti B, et al. Atmospheric pressure influences the risk of pneumothorax: beware of the storm! Chest 2007;131:1877-82. 